INPLASY

PROTOCOL

To cite: Ji et al. Prognostic value of microRNA-21 in epithelial ovarian carcinoma: a systematic review and metaanalysis protocol. Inplasy protocol 2020110064. doi: 10.37766/inplasy2020.11.0064

Received: 16 November 2020

Published: 16 November 2020

Corresponding author: Hongwei Wen

szycwhw@126.com

Author Affiliation:

Liaocheng People's Hospital

Support: ZR2016HB17.

Review Stage at time of this submission: The review has not yet started.

Conflicts of interest:

The authors declare that they have no competing interests.

\section{Prognostic value of microRNA-21 in epithelial ovarian carcinoma: a systematic review and meta-analysis protocol}

$$
\text { Ji, K1 Wang, XH²; Zhang, AQ3; Wen, } \mathrm{HW}^{4} \text {. }
$$

Review question / Objective: Whether the high expression of microRNA-21 is in association with poor prognosis in patients with epithelial ovarian carcinoma (EOC)?

Condition being studied: The expression of microRNA-21 has been shown to be associated with the prognosis in patients with malignant tumors. However, its prognostic value in epithelial ovarian carcinoma (EOC) remains controversial. This meta-analysis aimed to synthesize available data to clarify the association between microRNA-21 expression levels and clinical prognosis in EOC patients.

INPLASY registration number: This protocol was registered with the International Platform of Registered Systematic Review and Meta-Analysis Protocols (INPLASY) on 16 November 2020 and was last updated on 16 November 2020 (registration number INPLASY2020110064).

\section{INTRODUCTION}

Review question / Objective: Whether the high expression of microRNA-21 is in association with poor prognosis in patients with epithelial ovarian carcinoma (EOC)?

Rationale: MicroRNA-21 and epithelial ovarian carcinoma.
Condition being studied: The expression of microRNA-21 has been shown to be associated with the prognosis in patients with malignant tumors. However, its prognostic value in epithelial ovarian carcinoma (EOC) remains controversial. This meta-analysis aimed to synthesize available data to clarify the association between microRNA-21 expression levels and clinical prognosis in EOC patients. 


\section{METHODS}

Search strategy: The retrieval strategy will be created based on discussion of all the researchers on the basis of the Cochrane handbook guidelines. The plan searched terms are as follows: "ovarian carcinoma" or "epithelial ovarian carcinoma" or "oophoroma" or "OC" or "EOC" and "microRNA-21" or "miR-21" and "prognostic" or "survival". The detailed sample of search strategy for PubMed database is shown in Table 1. Similar search strategies will be modified and used for the other databases.

Participant or population: Patients must be diagnosed with EOC based on pathology and histology. No restrictions regarding age, gender, racial, region, education and economic status in this analysis. Patients with other malignancies are not included.

Intervention: In the experimental group, serum microRNA-21 expression levels were detected in all EOC patients confirmed by histopathology.

Comparator: In the control group, the expression levels of serum microRNA-21 were detected in normal participants.

Study designs to be included: All available clinical trials that assessed the effect of high expression of microRNA-21 on overall survival (OS) and disease-free survival (DFS) of patients with EOC will be included in this systematic review. The included studies should provide the relationship between miRNA-21 expression and clinical pathological characteristics.

Eligibility criteria: This study will include controlled clinical trials that assessed the effect of high expression of microRNA-21 on overall survival (OS) and disease-free survival (DFS) of patients with EOC. Articles without sufficient available data, animal experiments, case reports and series, literature reviews, meta-analysis, letters, conference abstract, and other unrelated studies will be all excluded from analysis.
Information sources: Electronic databases including Embase, Google Scholar, PubMed, Web of Science, Medline, Cochrane Library, China Scientific Journal Database, China National Knowledge Infrastructure, Chinese BioMedical Database and Wanfang Database, will be systematically searched for eligible literatures from their inception to November 2020. Language is limited with English and Chinese.

Main outcome(s): The primary outcomes will include: (i) Overall survival (OS, which is defined as the time from the date of randomization to death from any cause); (ii) Disease-free survival (DFS, which is the time from date of random assignment to date of recurrence or death); (iii) Hazard ratios (HRs) with corresponding 95\% confidence intervals (Cls) will be extracted from trials or be estimated from KaplanMeier survival curves by established methods.

Data management: Two investigators (Kun $\mathrm{Ji}$ and Xiaohua Wang) will be responsible for the data extraction independently. The following data will be extracted from eligible literatures. Study characteristics: first author's name, year of publication, country of study, sample size, microRNA-21 detection method, et al; Participant characteristics: age, gender, race, tumor stage, inclusion and exclusion criteria, et al; Outcome and other data: OS, DFS, HRs with corresponding $95 \%$ Cls, et al. When any data are missing or insufficient, we will contact original authors by using email. If the data is not available, we will only analyze the currently available data and discuss its potential impact.

Quality assessment / Risk of bias analysis: Two experienced authors (Kun $\mathrm{Ji}$ and Xiaohua Wang) will assess the risk of bias for each eligible study by using the Newcastle-Ottawa Quality Assessment Scale (NOS) independently. NOS comprise three quality parameters including selection, comparability, and result evaluation. Each study was scored from 0 to 9 according to these parameters, and $\geq 7$ were judged to be of higher quality. Any 
disagreements will be resolved via discussion with a third researcher (Anqi Zhang).

Strategy of data synthesis: Stata 14.0 (Stata Corp., College Station, TX, USA) and Review Manager 5.3 (Nordic Cochran Centre, Copenhagen, Denmark) statistical software were used for statistical analyses. Cochran's $Q$ and Higgins 12 statistic were used to assess heterogeneity among the included clinical trials. $\mathrm{P}<0.1$ for the Chi2 statistic or an $12>50 \%$ will be considered as showing considerable heterogeneity. A fixed effect model will be used to calculate the outcomes when statistical heterogeneity is absent; otherwise, the random effects model will be used for analysis. Odds ratio (OR) or HRs with corresponding $95 \% \mathrm{Cls}$ was used to evaluate the relationship between microRNA-21 expression and OS and DFS.

Subgroup analysis: Subgroup analysis will only be performed if sufficient clinical data is available. It will be conducted to explore the source of heterogeneity based on different race, EOC stages, microRNA-21 detection method, and survival data source.

Sensibility analysis: Sensitivity analysis of each parameter was carried out by one-byone elimination method to assess the reliability and robustness of the aggregation results. A summary table will report the results of the sensitivity analyses.

Language: Language is limited with English and Chinese.

\section{Country(ies) involved: China.}

Other relevant information: (i) Publication bias analysis: If the included studies are sufficient ( $\geq 10$ trials), we will detect publication biases of included trials using funnel plots, Begg's and Egger regression test. If publication bias existed, a trim-andfill method should be applied to adjust the pooled OR. (ii) Evidence evaluation: The guidelines of the Grading of Recommendations, Assessment, Develop- ment, and Evaluation (GRADE) will be used to assess the quality of evidence and the strength of the main result recommendations.

Keywords: miRNA-21, epithelial ovarian carcinoma, meta-analysis, prognosis.

Dissemination plans: We will disseminate the results of this systematic review by publishing the manuscript in a peerreviewed journal or presenting the findings at a relevant conference.

Contributions of each author:

Author 1 - Kun Ji - Conceptualization, Data curation, Formal analysis, Investigation, Methodology, Resources, Software, Supervision, Visualization, Writing-original draft.

Author 2 - Xiaohua Wang - Data curation, Formal analysis, Investigation, Methodology, Visualization, Writing-original draft.

Author 3 - Anqi Zhang - Formal analysis, Funding acquisition, Investigation, Methodology, Validation, Writing-review \& editing.

Author 4 - Hongwei Wen - Conceptualization, Project administration, Resources, Software, Supervision, Validation, Writingreview \& editing. 